\title{
The Influence of Selected Risk Factors on the Hearing Threshold Level of Noise Exposed Employees
}

\author{
Adam DUDAREWICZ ${ }^{(1)}$, Esko TOPPILA ${ }^{(2)}$, \\ Małgorzata PAWLACZYK-€USZCZYŃSKA ${ }^{(1)}$, \\ Mariola ŚLIWIŃSKA-KOWALSKA ${ }^{(1)}$ \\ (1) Nofer Institute of Occupational Medicine \\ Department of Physical Hazards \\ Św. Teresy 8, 91-348 Łódź, Poland \\ e-mail: adudar9999@wp.pl, \\ mpawlusz@imp.lodz.pl \\ (2) Institute of Occupational Health \\ Department of Physics \\ Topeliuksenkatu 41 a, FI-00250, Helsinki, Finland \\ (received May 5, 2010; accepted May 18, 2010)
}

The aim of the study was to evaluate the combined effect of noise exposure and additional risk factors on permanent hearing threshold shift. Three additional risk factors were: exposure to organic solvents, smoking and elevated blood pressure.

The data on exposure and health status of employees were collected in 24 factories. The study group comprised of 3741 noise male exposed workers of: mean age $39 \pm 8$ years, mean tenure $16 \pm 7$ years and $L_{E X, 8 \mathrm{~h}}=86 \pm 5 \mathrm{~dB}$. For each subject, hearing level was measured with pure tone audiometry, blood pressure and noise exposure were assessed from the records of local occupational health care and obligatory noise measurements performed by employers. Smoking and solvent exposure were assessed with questionnaire. The study group was divided into subgroups with respect to the considered risk factors. In the analysis, the distribution of hearing level of each subgroup was compared to the predicted one which the standard calculation method described in ISO 1999:1990. For each of the considered risk factors, the difference between measured and calculated hearing level distribution was used to establish, by the least square method, a noise dose related correction square function for the standard method. The considered risk factors: solvent exposure, smoking and elevated blood pressure combined with noise exposure, may increase degree of hearing loss.

Keywords: noise-induced hearing loss, risk factors. 


\section{Introduction}

In 2003 the European union introduced the noise directive, 2003/10/EC $D I$ RECTIVE 2003/10/EC OF THE EUROPEAN PARLIAMENT AND OF THE COUNCIL of 6 February 2003, on the minimum health and safety requirements regarding the exposure of workers to the risks arising from physical agents (noise), to reduce the harmful effects of noise. In the directive there are lower exposure action levels, $L_{E X, 8 \mathrm{~h}}=80 \mathrm{~dB}$ and $L_{C \text { peak }}=135 \mathrm{~dB}$, upper exposure action values $L_{E X, 8 \mathrm{~h}}=85 \mathrm{~dB}$ and $L_{C \text { peak }}=137 \mathrm{~dB}$ and limit values $L_{E X, 8 \mathrm{~h}}=87 \mathrm{~dB}$ and $L_{C \text { peak }}=140 \mathrm{~dB}$. In addition to the exposure limit values of the directive sets, many new requirements that have to be taken into account in the risk assessment: any effects concerning the health and safety of workers belonging to particularly sensitive risk groups; effects on workers' health and safety resulting from interactions between noise and work-related ototoxic substances.

The noise-induced hearing loss is related to life-time exposure, i.e. the life-time $A$-weighted sound energy ISO 1999 (1990). Although the present exposure can be evaluated reliably according to ISO 9612, knowledge of the previous exposure periods is often missing.

There is evidence from epidemiological studies that exposure to organic solvent mixture is associated with an increased risk of hearing loss. Organic solvents have detrimental effects both on the peripheral and central parts of the auditory pathway (ŚLIWIŃSKA-KOWALSKA, 2007). Styrene exposure combined with noise produces worse pure tone audiometric thresholds. Workers exposed to styrene and noise have poorer mean thresholds at 2, 3, 4 and $6 \mathrm{kHz}$, at the same time they have poorer distortion product otoacoustic emissions (DPOAE) (PRASHER et al., 2009). Mixtures of styrene with other solvents, such as toluene and n-hexane, increases the risk for hearing loss (ŚLIWIŃSKA-KOWALSKA et al., 2003; JOHNSON, 2007). The interaction between solvents and noise on the risk of hearing loss is unclear.

There is a positive association between smoking and hearing loss. The joint influence of noise and smoking on hearing status was reported by (Mizoue et al., 2003; Dengerink et al., 1992). Earlier studies suggested that combined with noise exposure, causes more adverse effect on hearing. Smoking can accelerate noise-induced hearing loss, but it is not clear whether the joint effect of noise and smoking is synergistic (Virokannas, AntTonen, 1995; Starck et al., 1999) or additive (MizOue et al., 2003; YCHIDA et al., 2005).

The elevated blood pressure may affect the regeneration mechanisms of ear. In the case of elevated blood pressure, the investigations indicate excess risks of hypertension related to long-term noise exposure (JARUP et al., 2008). There are many studies on noise and heart diseases. The comparison of the study results (VIRKKUNEN et al., 2005) confirms a slight increase of cardiovascular disease risk in populations exposed to work noise. The comparison can lead to the conclusion that noise exposure can contribute to the prevalence of cardiovascular disease, 
the evidence for a relation between noise exposure and ischemic heart disease is still inconclusive. Other study found that the female workers with low artery compliance or with high blood pressure might be suffering from hearing loss; those with noise-induced hearing loss might be suffering from hypertension if she is constantly exposed to loud industrial noise (Ni et al., 2007).

In properly screened hearing loss data, the two major causes exists: presbyacusis and noise exposure (ISO 1999, 1990). These two factors are strongly correlated and presbyacusis may even overrule the effect of noise in hearing loss. As a consequence, extremely large databases are needed, especially when combined effects are quantified. This is the major reason why no dose-relationships were created (ŚLIWIŃSKA-KOWALSKA et al., 2003). Luckily, the ISO 1999 (1990) provides a mathematical model for presbyacusis. Applying this model to eliminate the effect of presbyacusis reduces the number of subjects substantially. The purpose of this study is to evaluate the safe limits for noise exposure in presence of selected risk factors, using mathematical modeling.

\section{Material}

The data consisting of totally 4668 subjects from seven types of industries. For each subject, the pure tone audiometry of $1,2,3,4,6$ and $8 \mathrm{kHz}$ was recorded by the local occupational health care unit. The noise exposure was measured according to the procedure equivalent to ISO 9612-1997. For each worker, the mean daily average noise exposure $\left(\overline{L_{E X, 8 \mathrm{~h}}}\right)$ over all work periods was calculated using the measurement of all jobs (up to 8 jobs), when working for the current employer and the individual life-time noise exposures $\left(L_{i m}\right)$ are calculated under the assumption that workers were exposed to same noise exposure during work for previous employers. For $L_{i m}$ calculations, the following formulas were applied:

$$
\overline{L_{E X, 8 \mathrm{~h}}}=10 \lg \left(\frac{1}{\sum_{i=1}^{N} T_{i}} \sum_{i=1}^{N} T_{i} \times 10^{0.1 L_{E X_{i}}}\right)
$$

and

$$
L_{i m}=\overline{L_{E X, 8 \mathrm{~h}}}+10 \log (T),
$$

where $N$ is the total number of various time intervals/workplaces/jobs; $L_{E X i}$ is the equivalent continuous $A$-weighted sound pressure level normalized to a nominal 8 hour working day in the time interval/workplace/job $i$, in $\mathrm{dB} ; T_{i}$ is the duration of time interval $i$, in years; $T$ is total time in years of noise exposure.

The following data were collected: age, gender, tenure, cigarette smoking (years and numbers), head injury, history of acoustic trauma, ear diseases and operations, dizziness, vertigo and tinnitus, blood pressure, weight and length and 
exposure to organic solvents (styrene, toluene, xylene). In addition, questions on present and previous employment exposure to solvents and noise, exposure to risk factors during non-occupational activities, life style and medical history. In the case of lack of measurement results, the exposure to solvents and occurrence of other risk factors were rated qualitatively (yes/no).

In the case of the power plant and coal mine subgroup, the hygienic (sanitary inspection) records and medical files were explored and available data were introduced into the data base. Missing information was derived from questionnaire in $30 \%$ of the investigated cases.

2694 records were collected in the power plant and coal mine, these workers were exposed to noise at the workplace, aged from 22 to 63 years (mean $40 \pm 7$ ), the time of exposure (tenure) differed from 1 year to 40 years (mean $17 \pm 6$ ), and the individual of noise exposure levels related to 8 hours working day, averaged over exposure time, ranged from $70 \mathrm{~dB}$ to $91 \mathrm{~dB}(\mathrm{~A})$; mean $\overline{L_{E X, 8 \mathrm{~h}}}=84 \pm 4 \mathrm{~dB}$. The next 1047 records confine information considering male employees of lacquer factories, ship and yacht yards, plastic factories, processing industry and offices in this group the age changes from 20 to 61 years (mean $37 \pm 10$ ), the periods of exposure differed from 1 year to 42 years (mean 13 \pm 10 ) and individual of noise exposure levels related to 8 hours working day averaged over exposure time ranged from $71 \mathrm{~dB}(\mathrm{~A})$ to $100 \mathrm{~dB}(\mathrm{~A})$; mean $\overline{L_{E X, 8 \mathrm{~h}}}=89 \pm 7.0 \mathrm{~dB}$.

From the analysis, subjects meeting the following exclusion criteria were excluded. Subjects with audiogram not typical to noise-induced hearing loss NIHL. For these reasons 589, of which 122 subjects had an asymmetric hearing loss, subjects were excluded. Incomplete or possibly incomplete noise exposure history. Due to missing information about the exposure when working with previous employers, 375 subjects were excluded. Due to a small number of female, only men with at least one year of exposure to noise were taken into analysis. After these exclusions and validation, the database is comprised of 3741 male employees with mean age $39 \pm 8$ years, mean exposure time of $16 \pm 7$ years and $\overline{L_{E X, 8 \mathrm{~h}}}=88 \pm 5 \mathrm{~dB}$.

\section{Methods}

To examine the influence of noise and the potentially dangerous for hearing system risk factors as exposure to chemical agents, smoking and elevated blood pressure, the database was divided into subgroups of workers exposed to noise only and noise exposed combined with all the combinations of the selected risk factors, see Table 1. Subjects were classified for appropriate subgroups according to the following rules: exposure to solvents during occupational or nonoccupational activities (yes/no), tobacco smoking current users or non-users and subjects with low or elevated blood pressure meeting condition: (diastolic pressure) $>357 \mathrm{mmHg}-1 / 0.45$ (systolic pressure). 
Table 1. Mean ages, length of noise exposure, noise exposure levels and life-time noise exposure $L_{i m}$ of workers of the investigated risk subgroups (male subjects).

\begin{tabular}{|l|c|c|c|c|c|}
\hline \multirow{2}{*}{ Subgroup exposed to } & $\begin{array}{c}\text { Age } \\
\text { [years] }\end{array}$ & $\begin{array}{c}\text { Exposure } \\
\text { length } \\
\text { [years] }\end{array}$ & $\begin{array}{c}\text { Noise } \\
\text { exposure } \\
\text { level } \\
{[\mathrm{dB}]}\end{array}$ & $\begin{array}{c}L_{i m} \\
{[\mathrm{~dB}]}\end{array}$ & $\begin{array}{c}\text { Number } \\
\text { of cases }\end{array}$ \\
\cline { 2 - 6 } & \multicolumn{5}{|c|}{ Mean $\pm \mathrm{SD}$} \\
\hline Noise & $38 \pm 7$ & $17 \pm 7$ & $85 \pm 4$ & $97 \pm 5$ & 928 \\
\hline Noise and solvents & $35 \pm 9$ & $11 \pm 8$ & $87 \pm 7$ & $98 \pm 5$ & 304 \\
\hline Noise and smoking & $39 \pm 8$ & $18 \pm 7$ & $88 \pm 5$ & $97 \pm 8$ & 606 \\
\hline Noise and elevated blood pressure & $41 \pm 7$ & $18 \pm 6$ & $84 \pm 4$ & $97 \pm 4$ & 806 \\
\hline Noise and multiple risks & $38 \pm 6$ & $15 \pm 5$ & $86 \pm 5$ & $97 \pm 5$ & 1097 \\
\hline All male subjects & $39 \pm 8$ & $16 \pm 7$ & $86 \pm 5$ & $97 \pm 5$ & 3741 \\
\hline
\end{tabular}

To investigate of the influence of combination of noise and each of selected risk factors on hearing, the first thing to do is to remove the effect of presbyacusis. The following subgroups were formed: workers exposed to noise and only one of selected risk factors - i.e. "single risk factor subgroups". For each risk factor the analysis was performed in the same way. Details of analysis described below considered only subjects exposed to noise or exposed to noise and solvents. The basic features of the chosen subgroups are presented in Table 1.

The removed of the effect of age ISO 1999 (1990) model was used. For each above-mentioned subgroup, the deciles $(Q)$ of HL distribution of given by the standard ISO 1999 (1990) and measured, were found with respect to life-time noise exposure $\left(L_{i m}\right)$. To smooth the relationship between $Q$ of HL distribution and $L_{i m}$ values, the distribution were found for each $5 \mathrm{~dB}$ wide intervals of $L_{i m}$, moved with $1 \mathrm{~dB}$ step from the bottom to the upper range of the $L_{i m}$. Simultaneously, the number of cases $(n)$ and average values of the $L_{i m}$ within each position of the mowing $5 \mathrm{~dB}$ interval were found. In this way, relations between $L_{i m}$ and values of the observed and predicted HL for considered deciles of the HL distribution were obtained. The same procedure was repeated for HL at $1,2,3,4$ and $6 \mathrm{kHz}$.

The HL values of the observed deciles differ from the predicted ones and these differences are related to $L_{i m}$ and $(Q)$. To describe the difference between the observed and predicted distribution in examined subgroups, the second degree polynomial functions were fitted. In the fitting, a weight $w=n^{0.5}$ was applied to each point, where the observed and predicted HL distributions were found. The difference function $D F_{s}\left(L_{i m}, Q\right)$ for noise and solvent exposed subgroup and noise exposed subgroup were calculated and functions are expressed by the following equation:

$$
\begin{aligned}
D F_{s}\left(L_{i m}, Q\right)= & \operatorname{const}_{s}+A_{s} \cdot L_{i m}+B_{s} \cdot(100-Q)^{2} \\
& +C_{s} \cdot L_{i m} \cdot(100-Q)+D_{s} \cdot L_{i m}+E_{s} \cdot(100-Q),
\end{aligned}
$$


where: $Q$ are the deciles corresponding to the ISO 1999 convention of fractiles, equal to $(10 ; 20 ; \ldots ; 90)$, in $\% ; L_{i m}$ - is the life-time noise exposure, in $\mathrm{dB}$, Const, $A_{s}, B_{s}, C_{s}, D_{s} ; E_{s}$ and Const - are parameters depending on frequency and risk subgroups (noise exposed, noise and chemical exposed).

It was assumed that the combined exposure to noise and solvents in one of the considered subgroups leads to difference between the founded $C F_{s}$ functions. The $C F_{\text {chem }}$ function relates the difference between founded $D F_{s}$ functions with life-time exposure to noise and deciles of the HL distribution $\left(L_{i m}, Q\right)$ :

$$
C F_{\text {chem. }}\left(L_{i m}, Q\right)=D F_{\text {noise } \& \text { chem }}\left(L_{i m}, Q\right)-D F_{\text {noise }}\left(L_{i m}, Q\right) \text {. }
$$

In the same form as the one presented by Eq. (3) with modified parameters, the function $C F_{\text {chem }}$ was found with no weighted fitting procedure. The function $C F_{\text {chem }}$ describes the deviation of the observed HL distribution of subjects exposed to combined exposure to noise and chemical agents from the standard ISO 1999 model prediction. This means that all the deciles $Q$ of the HL distribution calculated with standard model for considered $L_{i m}$ should be corrected by adding penalties equal to values of the $C F_{\text {chem }}$ function at the same $L_{i m}$ and $Q$. As the predictions of the HL for different subjects depend on $L_{i m}$ and subjects' age, the total distributions of predicted HL were build by summarizing the predicted HL distribution of each subject. Total distribution calculated with standard ISO 1999 and ISO corrected models were compared with the observed HL distribution in examined subgroups.

The correction functions were used to calculate the HL predicted distribution in the whole study group - including subjects exposed to more than one risk factor. The appropriate penalties were applied for ISO 1999 model prediction with respect to the subjects' risk factors. Distribution of measured HL and predicted according to ISO 1999 model and ISO model with corrections were compared with statistical tests.

\section{Data analysis}

The calculation and data analysis were performed with (StatSoft, Inc. (2004). STATISTICA (data analysis software system), version 6. www.statsoft.com). The functions were founded with the least square method. Observed and predicted HL distribution were compared using the Kolmogorov Smirnov test and nonparametric Mann Whitney U test.

\section{Results}

In spite of the observed life-time noise exposure depending differences between the measured and ISO 1999 predicted hearing threshold level (HL) at all frequencies 1, 2, 3, 4 and $6 \mathrm{kHz}$, the correction functions for noise and chemical exposure $C F_{\text {chem }}$ were found for $2,3,4$ and $6 \mathrm{kHz}$. Due to the fact that the 
hearing losses are distributed in quite narrow range at first three investigated frequencies, especially at $1 \mathrm{kHz}$, only $86 \%$ of cases have HL smaller than $20 \mathrm{~dB}$, the fitting procedure could not be applied at $1 \mathrm{kHz}$. At 2 and $3 \mathrm{kHz}$, the correction functions were founded with fitting procedure applied for small deciles $Q$ of HL distribution.

Table 2 presents the coefficients of fitted correction functions at 2, 3, 4 and $6 \mathrm{kHz}$ with intervals of the $L_{i m}$ and $Q$, where fitting procedure was performed. This was caused by the very uncertain results for HL predictions and measurements of values les than $10 \mathrm{~dB}$. Due to these limitations the correction functions at frequencies 2 and 3 should be considered just as an approximation for deciles above 50 at frequencies 2 and $3 \mathrm{kHz}$; the same might be told on deciles above $Q=70$ at frequencies 4 and $6 \mathrm{kHz}$.

The predicted HL for noise and solvent exposed group were calculated at 3 , 4 and $6 \mathrm{kHz}$. Figures $1 \mathrm{a}$ and $1 \mathrm{~b}$ show comparison between the measured and predicted distribution of hearing losses at 4 and $6 \mathrm{kHz}$ as a function of life time exposure. The drawn deciles are 10,50 and 90. It may be assumed that calculation with standard ISO 1999 method describe noise-induced hearing loss due to noise exposure, while ISO with corrections shows hearing loss caused by combining exposure to noise and chemical agents.

The medians and widths of the measured HL distribution at 3 and $6 \mathrm{kHz}$ (marked with points and whiskers showing deciles obtained from the data, Fig. 1b), remain quite stable trough the whole range of $L_{i m}$ life-time exposures. This is due to the fact that the subjects in the upper end, exposure over $100 \mathrm{~dB}$, they were relatively young and had the highest daily exposures of all populations, majority of them were working as painters in shipyards. There are small elevations of the measured HL distribution visible at the lower end of $L_{i m}$ ranges (probably because of underestimation of $L_{i m}$, non-occupational noise exposure may be a significant in the case of low $L_{i m}$ values).

At $3 \mathrm{kHz}$, there are no differences between both the predicted HL distribution up to $L_{i m}=96 \mathrm{~dB}$. Above this value, the differences of $10 \%$ deciles start to increase up to $15 \mathrm{~dB}$. Differences between medians appear for $L_{i m}>100 \mathrm{~dB}$.

Comparison of HL predicted with both methods shows constant shift at 4 and $6 \mathrm{kHz}$, when the Lim life time exposure is small, below $96 \mathrm{~dB}$.

At $4 \mathrm{kHz}$ (Fig. 1a), the median corrected due to chemical exposure, is shifted $\sim 5 \mathrm{~dB}$ upwards and the $10 \%$ decile about $\sim 10 \mathrm{~dB}$. This shift is due to the pure ototoxic effect of the solvents. When the life-time exposure exceeds 95-96 dB, the distribution becomes wider and the median value of HL increases; at lifetime exposure of 104-105 dB the median HL is $\sim 20 \mathrm{~dB}$ higher than predicted by the ISO model and the difference of $10 \%$ deciles at $104-105 \mathrm{~dB}$ is $\sim 30 \mathrm{~dB}$.

At $6 \mathrm{kHz}$ (Fig. 1b) there is a constant $\sim 10 \mathrm{~dB}$ shift between medians of both predicted HL, the shift is independent of the $L_{i m}$ value. Similar shift can be seen for $10 \%$ deciles when the $L_{i m}$ is below $96 \mathrm{~dB}$; with increasing $L_{i m}$ the shift increases up to $\sim 25 \mathrm{~dB}$. 


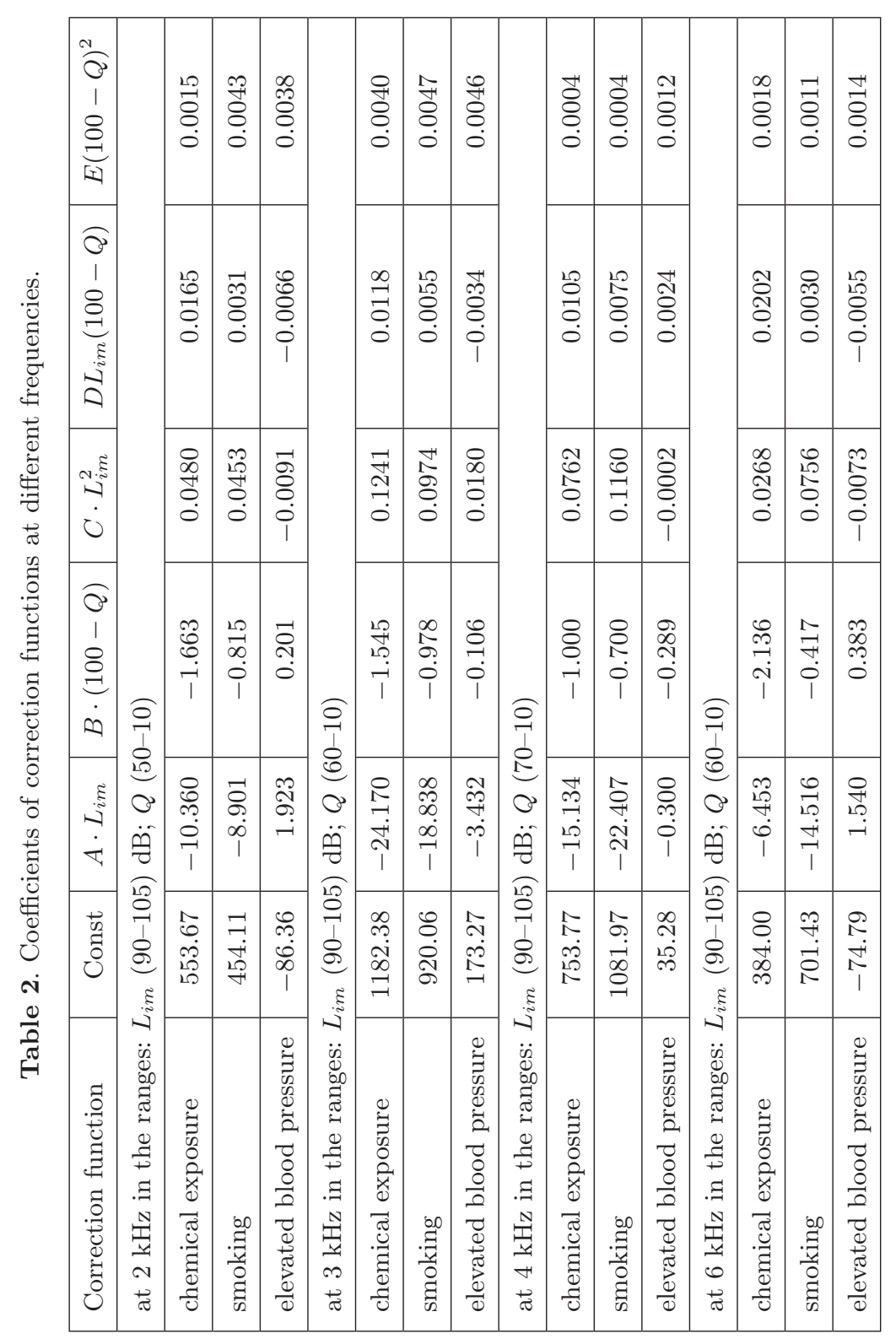


a)

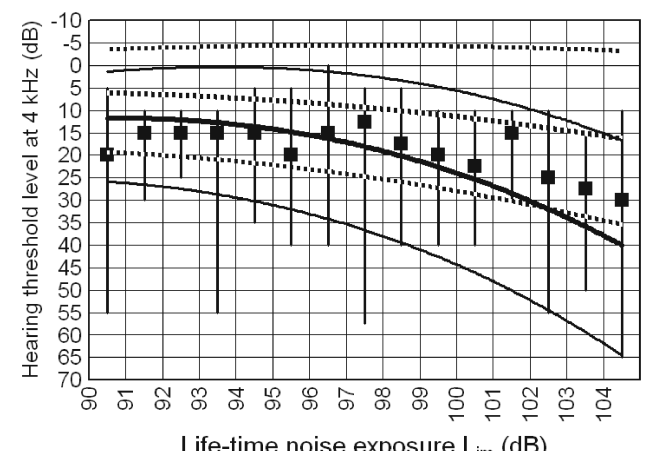

b)

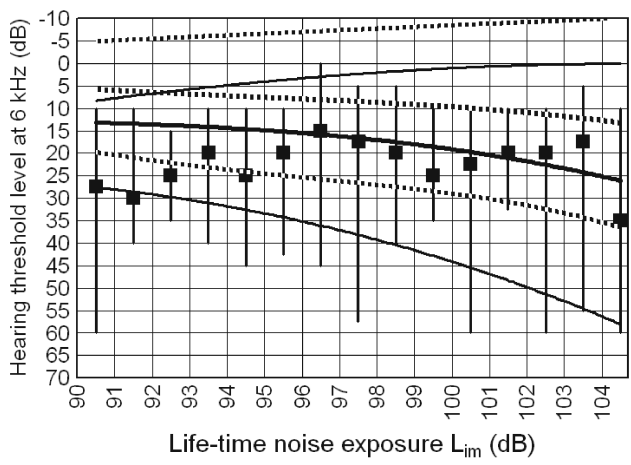

Fig. 1. Deciles 10, 50, 90 of the HL distribution as a function of $\mathrm{L}_{i m}$ at 4 and $6 \mathrm{kHz}$; measured (points) and predicted according to ISO 1999 (dashed lines) and ISO corrected model (solid lines).

a)

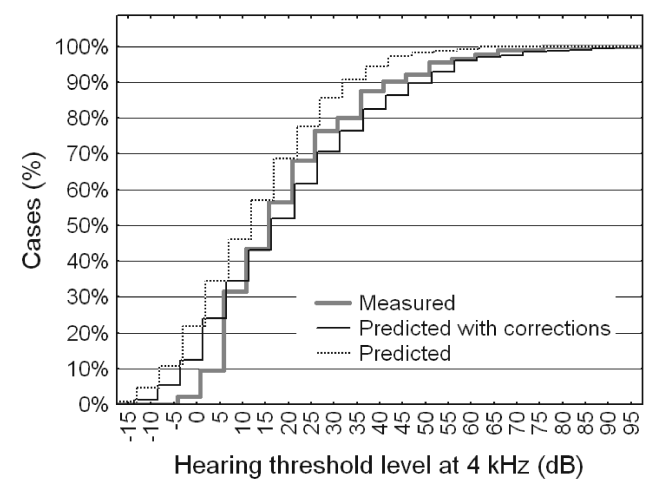

b)

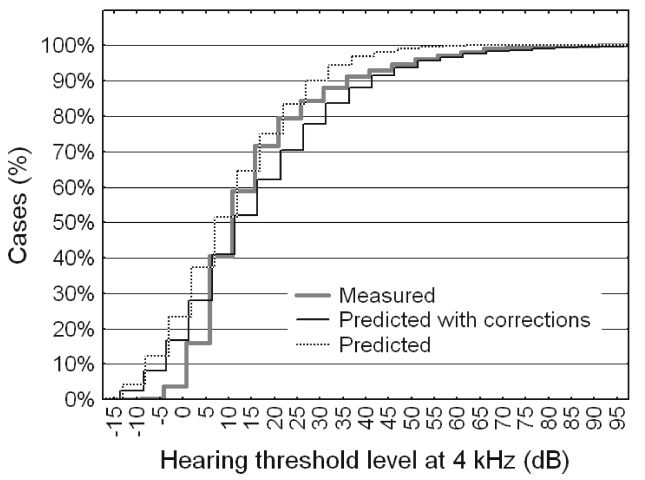

Fig. 2. Cumulative distribution of HL, measured and calculated according to ISO 1999 model and ISO with corrections: a) noise and solvent exposed, b) whole study group.

Figure 2a presents cumulative HL distribution in noise and solvent-exposed subgroup (only exposure to solvent) and (Fig. 2b) presents HL distribution in whole study group. It was found that predicted HL distributions are closer to the measured ones especially for HL greater than $35 \mathrm{~dB}$.

The results showed that elevated blood pressure increased the hearing loss but the increase seems to be independent of the noise exposure. For smoking there seems to exist an effect combined with noise. The effect seems to start when $L_{i m}$ exceed $96 \mathrm{~dB}$.

\section{Discussion}

The 2003/10/EC noise directive sets the lower action limit value to $L_{E X, 8 \mathrm{~h}}=$ $80 \mathrm{~dB}$. Under this value no hearing loss can occur at speech frequencies $\left(0.5^{-}\right.$ 
$2 \mathrm{kHz}$ ) according to ISO 1999, but for high frequencies it is possible. In our study, the combined effect seems to start when the life time exposure $L_{i m}>96 \mathrm{~dB}$. This is equivalent to 20 years of daily exposure to $\overline{L_{E X, 8 \mathrm{~h}}}=83 \mathrm{~dB}(\mathrm{~A})$. From this point of view the lower action limit value seems to be appropriate. In this study we could not set a limit value for ototoxic exposure, because the accuracy of the chemical exposure is a more complicated issue and there is no good metrics for the life-time exposure. This is why we contented to divide the subjects into exposed and non-exposed.

The impact was highest at $4 \mathrm{kHz}$, not observable at $1 \mathrm{kHz}$ and small at $2 \mathrm{kHz}$. In literature, styrene causes a high frequency hearing loss in the region of $2-6 \mathrm{kHz}$ whereas mixtures affect the mid-frequencies, $1-2 \mathrm{kHz}$. This indicates that different solvent exposures may have different impact to the number of NIHL case.

ŚLIWIŃSKA-KOWALSKA et al. (2007) showed that the mean hearing thresholds at frequencies of $2-4 \mathrm{kHz}$ were poorer for workers exposed to solvents plus noise than for the solvent-only group. Our result confirms this. In addition, we are able to show that the combined effect starts to use when the life time exceeds $96 \mathrm{~dB}$.

Our results are also in good agreement with the results of the animal studies made by CAMPO and MAguin (2007). Their result is that daily exposure limit $80 \mathrm{~dB}$ is the threshold value below which no combined effect with styrene exist. Thus when a combined exposure to solvents and noise exist, the hearing protection should always start when daily exposure exceeds $80 \mathrm{~dB}$. Smoking and elevated blood pressure combined with noise exposure may increase the degree of hearing loss. The influence of smoking combined with noise exposure on hearing loss depend on $L_{i m}$ while in the case of elevated blood pressure and noise the influence is independent from $L_{i m}$.

\section{Conclusions}

Exposure to solvents has adverse effect on the hearing status. It enlarges hearing threshold levels by $5-10 \mathrm{~dB}$ at 4 and $6 \mathrm{kHz}$ even for life-time noise exposure $L_{i m}<96 \mathrm{~dB}$. Exposure to chemicals combined with noise exposure of higher levels causes greater hearing damages in comparison with the ones caused by noise. The damages increase with increasing life-time noise exposure level, combined exposure enlarged medians of HL distribution by $10-30 \mathrm{~dB}$ at $L_{i m}=$ $105 \mathrm{~dB}$. The effect is greater for sensitive persons. The combined effects between noise and solvents are negligible below the lower action limit value $L_{E X, 8 \mathrm{~h}}=$ $80 \mathrm{~dB}$ set by the $2003 / 10 / \mathrm{EC}$ noise directive. The influence of smoking combined with noise exposure on hearing loss depends on $L_{i m}$, while in the case of elevated blood pressure and noise the influence is independent of $L_{i m}$. 


\section{Acknowledgments}

This report was supported by the project of the 6th European Framework Project "NoiseHear" (Contract MTKD - CT - 2004 - 003137) and by the European Social Found in Poland NFSR for years 2007-2013 (Project WND POKL.02.03.01 - $00-001 / 08)$.

\section{References}

1. Campo P., Maguin K. (2007), Solvent-Induced Hearing Loss: Mechanisms and Prevention Strategy, Int. J. Occup. Med. Environ. Health, 20, 3, 265-270.

2. Dengerink H.A., Lindgren F.L., Axelsson A. (1992), The interaction of smoking and noise on temporary threshold shifts, Acta Otolaryngol, 112, 932-938.

3. ISO 1999 Acoustics - Determination of occupational noise exposure and estimation of noise induced hearing impairment. International Organization for Standardization, Geneva 1990.

4. Jarup L., Dudley M., Babisch W., Houthuijs D., Swart W., Pershagen G., Bluhm G., Katsouyanni K., Velonakis M., Cadum E., Vigna-Taglianti F. (2008), Hypertension and Exposure to Noise Near Airports: the HYENA Study, Environ Health Perspect., 116, 3, 329-333.

5. Johnson A. (2007), Relationship between styrene exposure and hearing loss: review of human studies, Int. J. Occup. Med. Environ. Health, 20, 4, 315-325.

6. Mizoue T., Miyamoto T., Shimizu T. (2003), Combined effect of smoking and occupational exposure to noise on hearing loss in steel factory workers, Occup. Environ. Med., $60,56-59$.

7. Ni C.-H. Chen Z.-Y., Zhou Y., Zhou J.-W., Jing-Jing P., Liu N., Wang J., LiAng C.-K., Zhang Z.-Z., Zhang Y.-J. (2007), Associations of blood pressure and arterial compliance with occupational noise exposure in female workers of textile mill, Chin Med. J. (Engl.), 120, 15, 1309-1313.

8. Prasher D. et al. (2009), Noise and Industrial Chemicals: Interaction Effects on Hearing and Balance NoiseChem. Key Action 4: Environment and Health. 2001-2004. FINAL REPORT, http://ec.europa.eu/research/quality-of-life/ka4/pdf/report_noisechem_en.pdf (20 Oct. 2009).

9. Starck J., Toppila E., PYykKo I. (1999), Smoking as a risk factor in sensory neural hearing loss among workers exposed to occupational noise, Acta Otolaryngol, 119, 302-305.

10. Śliwińska-Kowalska M. (2007), Exposure to organic solvent mixtures and hearing loss: literature overview, Int. J. Occup. Med. Environ. Health, 20, 4, 309-314.

11. Śliwińska-Kowalska M., Zamyseowska-Szmytke E., Szymczak W., KotyŁo P., Fiszer M., WesoŁowski W. et al. (2003), Ototoxic effects of occupational exposure to styrene and co-exposure to styrene and noise, J. Occup. Environ. Med., 45, 1, 15-24.

12. Virkkunen H., Kauppinen T., Tenkanen L. (2005), Long-term effect of occupational noise on the risk of coronary heart disease, Scand J. Work Environ. Health, 31, 4, 291-9. 
13. Virokannas H., Anttonen H. (1995), Dose-response relationship between smoking and impairment of hearing acuity in workers exposed to noise, Scand Audiol, 24, 211-216.

14. Ychida Y., Nakashimat T., Nino N., Shimokata H. (2005), Is there a relevant effect of noise and smoking on hearing? A population-based aging study, Int. J. Audiol., 44, $86-91$. 